\title{
Author response to "Scientific rigour is far more than a matter of sample size"
}

\author{
Michael W. Rabow
}

Received: 16 December 2014 / Accepted: 8 January 2015 / Published online: 18 January 2015

(C) Springer-Verlag Berlin Heidelberg 2015

Although we appreciate the comments offered by Pär Salander, a number of points require clarification. The limitations of our study were described explicitly in the published discussion of our results. Nowhere do we suggest that a large sample size is a substitute for scientific rigor. Our study used a convenience sample to begin to explore an important but understudied element of clinical palliative care.

First, we did not suggest that our analysis could serve as the final assessment of spirituality in palliative care consultation, but believe that our data can serve as a useful continuation of the scant current literature. Regarding the correlation between quality of life and spirituality, although these outcomes are clearly related to "how people are doing," they point to specific elements of this very broad element of human experience. That they are appropriately categorized within the same expansive human experience does not necessarily suggest that they are the same concept underserving of comparative analysis. There clearly are situations, depending on an individual's values and focus, when some patients might experience a good quality of life overall but have limited spiritual wellbeing. While our quantitative data are not adequate to explore the subtle details of the distinctions between symptoms, quality of life, and spiritual well-being, the correlation between the outcomes we presented does justify further research with the ability to perform logistic regressions to detail complex and subtle differences.
Second, although the letter's author is uncomfortable with reducing spirituality to a single screening question, this is the explicit goal of screening questions. We did, in fact, use a question validated to screen for both religious and existential well-being. While there is clearly further detail within these two domains of spirituality, the survey employed in our study has been demonstrated to be useful for the purposes we employed. Again, this study using a screening question calls for further research to get to the complexity not elucidated in the screen. Screening questions are designed to point to the need for further exploration.

Finally, and most importantly from my perspective, the author critiques the importance of this research, but completely ignores a discussion of the most valuable part of the work. The reviewer makes no comment about the association between the palliative care intervention and spirituality, writing "I will not dwell on the first part of the study." However, in ignoring the core of the study, the reviewer's dissatisfaction with the second part takes on inappropriate significance and discounts the key reason to share this data with other interested clinicians and researchers.

Michael W. Rabow, MD

Conflict of interest Dr. Michael W. Rabow declares that he has no competing financial interests.

Dr. Rabow has full control of the primary data and will allow the journal to review the data if requested.
M. W. Rabow $(\bowtie)$

School of Medicine, University of California, San Francisco, San

Francisco, CA, USA

e-mail:mrabow@medicine.ucsf.edu 